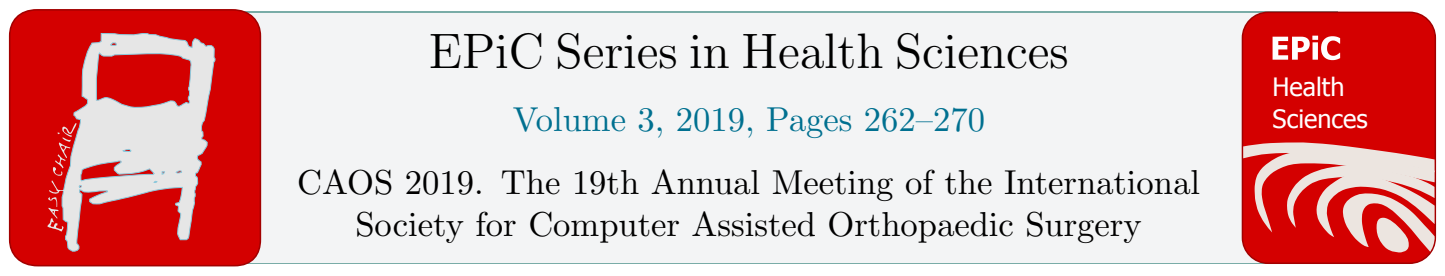

\title{
Accuracy of Image-less Navigation for Functional Cup Positioning in Total Hip Arthroplasty
}

\author{
Morteza Meftah $^{1}$, Vinay H. Siddappa ${ }^{2}$, Jeffrey M. Muir ${ }^{3}$, Peter B. White ${ }^{4}$ \\ ${ }^{1}$ Division of Adult Reconstructive Surgery, NYU Langone Orthopaedic Hospital, \\ NYU Langone Health, New York, New York, USA \\ ${ }^{2}$ Adult Reconstruction Fellow, BronxCare Hospital Center, Bronx, New York, USA \\ ${ }^{3}$ Division of Clinical Research, Intellijoint Surgical, Waterloo, Ontario, Canada \\ ${ }^{4}$ Orthopaedic Surgery Resident, Northwell Health Plainview Hospital, \\ Plainview, New York, USA \\ Morteza.Meftah@NYUMC.org
}

\begin{abstract}
Computer-assisted navigation has the potential to improve the accuracy of cup positioning during total hip arthroplasty (THA) and prevent leg length discrepancy (LLD). The purpose of this study was to compare acetabular cup position and postoperative LLD after primary THA using posterolateral approach. Between August 2016 to December 2017, 57 THAs using imageless navigation were matched with 57 THA without navigation, based on age, gender and BMI. Post-operative weight-bearing radiographs were assessed using for anteversion, inclination and LLD. Goal for functional cup placement was $40^{\circ}$ inclination and $20^{\circ}$ anteversion based on preoperative weight bearing pelvic images. Functional LLD was measured as compared to preoperative radiographs and contralateral side. Proportion of cups within Lewinnek's safe zone, proximity to a pre-operative target of and the LLD $>5 \mathrm{~mm}$ was assessed. The mean age was $54.9 \pm 9.6$ years $(30-72)$ and $57.6 \pm 12.5$ years $(20-85)$ in control and navigated groups, respectively. Mean cup orientation in the navigated group was $20.6^{\circ} \pm 3.3^{\circ}(17-$ $25)$ of anteversion and $41.9^{\circ} \pm 4.8^{\circ}(30-51)$ of inclination, vs. $25.0^{\circ} \pm 11.1^{\circ}(10-31)$ and $45.7^{\circ} \pm 8.7^{\circ}(29-55)$ in control group, where were statistically significant $(\mathrm{p}=0.005$ and $\mathrm{p}=0.0001$ ), respectively. In the navigated group, significantly more acetabular cups were placed within Lewinnek's safe zone (anteversion: $78 \%$ vs. $47 \%, \mathrm{p}=0.005$; inclination: $92 \%$ vs. $67 \%, \mathrm{p}=0.002$ ). There was no significant difference in mean LLD in navigation and control groups $(3.1 \pm 1.5 \mathrm{~mm}$ vs. $4.6 \pm 3.4 \mathrm{~mm}, \mathrm{p}=0.36)$, although fewer LLDs $>5$ $\mathrm{mm}$ were reported in the navigated group $(7.1 \%)$ than in controls $(31.4 \%, \mathrm{p}=0.007)$. The use of this image-less computer-assisted navigation improved the accuracy with which acetabular cup components were placed and may represent an important method for limiting post-operative complications related to cup malpositioning and LLD.
\end{abstract}




\section{Introduction}

Total hip arthroplasty (THA) is among the most successful orthopaedic procedures [1]. However, despite an overall high rate of success, the potential for significant post-operative complications exists, the majority of which relate to inaccuracies in component placement [1-3]. In particular, malpositioning of the acetabular cup component is associated with instability, loosening, increased component wear and an increased risk of dislocation, all of which contribute to an increased likelihood of costly revision surgery [4-7]. Traditionally, surgeons rely on experience, use of anatomic landmarks or jigs to provide a basis for component placement. Acetabular cup components implanted using these conventional methods; however, are subject to patient positioning or finding anatomical landmarks, with some studies suggesting that up to $62 \%$ of conventionally implanted acetabular cup components are malpositioned [8].

Computer-assisted navigation systems have been developed in recent years to assist surgeons with the accurate placement of components during THA. These systems are generally associated with improved accuracy in component placement, with improvements in post-operative leg length, safe zone proportionality and proximity to pre-operative target noted across several studies comparing navigation with manual methods [9-14]. However, the drawbacks of such systems - high capital costs, potentially significant lengthening of procedural time - have limited their use to only a small fraction of THAs [15, 16]. Despite these limitations, there are certainly areas where navigation is superior to traditional methods, findings that warrant continued investigation of the ability of navigation to improve outcomes in THA.

The ongoing debate over the validity of Lewinnek's safe zone [17] and the increased interest in functional cup positioning, especially in patients with abnormal pelvic tilt during standing and sitting, such as lumbar fusion that directly impact hip mobility, have created an opportunity for navigation to have an important impact on post-operative outcomes. As the focus of pre-operative planning shifts to a more patient-specific orientation, the proximity of the post-operative position to the pre-operative target is of growing importance and is an area where navigation has shown promise [9, 11-13].

The purpose of our study was to evaluate the impact of an imageless navigation system to accurately achieving pre-operative targets for cup position and for leg length changes. Our hypothesis was that navigation assistance would be associated with a higher proportion of components implanted within Lewinnek's safe zone and an improved ability to achieve preoperative targets for acetabular cup position and change in leg length. 


\section{Methods}

This study was a retrospective review of our prospective database of primary THA procedures performed with the assistance of a novel, imageless computer-assisted navigation tool, compared with matched controls completed using traditional methods. Institutional Board Review approval was obtained prior to this study. Patients were eligible for inclusion if they underwent a primary unilateral THA procedure (modified MIS posterolateral approach) performed by the senior author (**) between August 2016 and December 2017. Two cohorts (navigation versus conventional) were included in this case-matched study. 57 patients from the navigation cohort were matched to 57 patients from the conventional cohort based on age, gender and BMI. In the intervention group, THA was performed with the assistance of the navigation tool (Intellijoint HIP ${ }^{\text {, , Intellijoint }}$ Surgical, Waterloo, ON). In the control group, THA was performed traditionally using mechanical guides and anatomical landmarks.

General inclusion criteria in the intervention group included the use of the navigation tool during the study period and the ability to obtain pre- and post-operative anteroposterior (AP) weight-bearing radiographs. Patients were excluded from the study if the navigation device was removed for any reason prior to final measurements being collected; if radiographic measurement landmarks on the pre- or post-operative imaging were not visible or able to be properly reproduced.

All procedures were performed via a modified MIS posterolateral surgical approach. The use of the navigation tool consists of a camera, magnetically attached to a platform fixed to the operative iliac crest via two surgical pins, and a tracker, magnetically attached to a platform fixed to the greater trochanter or to other objects (e.g. impactor) during surgery. The camera computes position and orientation of the tracker to assess changes in leg length and center of rotation. This data is sent to the computer workstation in real time. Utilization of this data has been described in detail elsewhere $[18,19]$. In the control group, acetabular cup components were implanted using traditional mechanical guides and anatomic landmarks [20].

Baseline data including demographic data (age, gender, BMI) was collected for all patients in both study groups. Patients were followed prospectively for 3 months post- operatively. Primary outcomes for this study included cup position (anteversion and inclination), functional leg length discrepancy (pre- to post-operative change in operative vs. non-operative leg length discrepancy), proportion of cup components placed within the Lewinnek safe zone [17], proportion of cups placed within $\pm 10^{\circ}$ of a pre-operative target of $40^{\circ}$ inclination $/ 20^{\circ}$ anteversion, proximity of final cup position to that pre-operative target and proportion of leg length differentials $>5 \mathrm{~mm}$.

Secondary outcomes included the post-operative rates of dislocation, hospital readmission, reoperation, and revision surgery. The rate of complications, including pain at the site of the pins/screws, was also recorded at follow-up visits.

Images were scaled using a standard $25.4 \mathrm{~mm}$ scaling object. In cases where no scaling object was available, post-operative images were scaled using the known diameter of the femoral head. Cup orientation and changes in leg length were calculated using TraumaCad (Brainlabs, Chicago, IL) and the inter-ischial line method [21]. Each image was read 3 times for intra-observer reliability and the results averaged to provide final values for leg length change and cup position. All images were reviewed by two independent, non-blinded observers $(* * * \& * * *)$.

Alpha was set a priori at 0.05 for all statistical comparisons. Mean values were compared using independent samples t-tests and/or single-factor ANOVA. Mean values are expressed as mean (standard deviation). Intra-rater reliability was evaluated using the intraclass correlation coefficient (ICC). 


\section{Results}

A total of 114 patients (intervention: 57, control: 57) were eligible for inclusion and included in the final analysis. The mean age was $54.9 \pm 9.6$ years $(30-72)$ and $57.6 \pm 12.5$ years $(20-85)$ in control and navigated groups, respectively. The mean BMI in control and navigated groups was $32.8 \pm 3.4$ (27$41)$ and $33.2 \pm 4.1(27-43)$, respectively. There was no difference mean age, operative side or gender between the intervention and control groups (Table I).

Mean functional anteversion in the navigated group was $20.6^{\circ} \pm 9.3^{\circ}$ (range: $17^{\circ}-25^{\circ}$ ), vs $25.0^{\circ} \pm 11.1^{\circ}$ (range: $10^{\circ}-31^{\circ}$ ) in the control group ( $\mathrm{p}=0.005$ ). Significantly more cups were placed within the Lewinnek safe zone in the navigation group (62/79, 78\%) when compared with controls $(44 / 93,47 \%, X 2=17.5, p<0.001)$. Similarly, a significantly greater proportion of cups were placed within the functional zone of $20^{\circ} \pm 10^{\circ}$ of anteversion in the navigated group vs. controls $(55 / 79(70 \%)$ vs. $49 / 93(53 \%), X 2=5.1, \mathrm{p}=0.02)$.

Mean inclination in the navigation group $\left(41.9^{\circ} \pm 4.8^{\circ}\right.$; range: $\left.\left.30^{\circ}-51^{\circ}\right)\right)$ was significantly lower than that of the control group $\left(45.7^{\circ} \pm 8.7^{\circ}\right.$; range: $\left.\left.29^{\circ}-55^{\circ}\right), p=0.01\right)$. A significantly greater proportion of acetabular cups were placed within the Lewinnek/functional safe zone $\left(40^{\circ} \pm 10^{\circ}\right)$ in the navigated group vs. controls (73/79 (92\%) vs. 62/93 (67\%), X2=16.8, $\mathrm{p}<0.001)$.

The mean post-operative leg length discrepancy in the navigation group $(3.1 \pm 1.5 \mathrm{~mm})$ was less than that of the control group $(4.6 \pm 3.4 \mathrm{~mm})$, although this finding was not statistically significant $(\mathrm{p}=0.36)$. Significantly fewer cases of $\mathrm{LLD}>5 \mathrm{~mm}$ were observed in the navigation group than in the control group ( $7 \%$ vs. $31 \%, \mathrm{X} 2=7.2, \mathrm{p}=0.007)$.

At final follow-up, there were no dislocation, fracture, re-operation or revisions. No reports of pain at the site of the pelvic pins in the intervention group at 90-days were reported. No other complications were reported.

Triplicate measurement of radiographic parameters was consistent, with strong intra-rater reliability for all measured parameters in both study groups (ICC: anteversion (intervention: 0.983, control: $0.984)$, inclination $(0.980,0.983)$ and leg length $(0.965,0.978))$.

\section{Discussion}

Total hip arthroplasty remains a successful procedure to address degenerative hip arthritis, although potential complications associated with component malpositioning and leg length discrepancy continue to present challenges to surgeons. The use of computer-assisted navigation has improved component accuracy, although has not been fully embraced by the orthopaedic community, as concerns over cost and potential time-add limit its acceptance. We evaluated the accuracy of component placement in a cohort of patients who underwent primary or revision THA with the assistance of an imageless navigation system, in comparison to matched controls who underwent THA using traditional methods.

Our study has limitations. Retrospective data collection may limit the strength of the conclusions; however, the use of a case-matched control group allows for a valid comparison of navigated data with traditional, non-navigated data and provides more comprehensive findings than would a non-controlled cohort study. Similarly, the retrospective collection of data limits our ability to analyze cases for changes in offset, as the variability between patient positioning on radiographs renders an analysis of offset inaccurate.

We found that there was a significant improvement in cup position in the navigated group, with fewer acetabular cups placed outside of the safe zone and fewer leg length differentials $>5 \mathrm{~mm}$. LLD represent one of the most common causes of litigation against orthopedic surgeons [22, 23], up to $15 \%$ of whom will face a new claim each year [24]. Patients are known to perceive LLDs that exceed $5 \mathrm{~mm}$ $[25,26]$, a threshold that many studies have demonstrated is difficult to achieve in non-navigated THA 
[27-30]. Indeed, many studies report proportion analyses involving LLD using thresholds of 6 or $7 \mathrm{~mm}$, potentially reflecting the limits of accuracy associated with more traditional THA methods of monitoring cup position and leg length [27, 28]. In our study, we noted a mean post-operative LLD of $3.1 \mathrm{~mm}$ in navigated cases and, while not significantly different from the non-navigation cases (mean: $4.6 \mathrm{~mm}$ ), the proportion of cases in which the LLD was $>5 \mathrm{~mm}$ was significantly lower in the navigated group $(7 \%$ vs $31 \%, p=0.007)$. This suggests a strong role for navigation in preventing post-operative LLDs following THA, which may be associated with potentially significant decreases in hospital costs. Evidence indicates that up to $1 / 3$ of cases brought against orthopaedic surgeons are settled, at an average cost of $\$ 35,651$, suggesting one avenue for potentially significant cost savings when utilizing navigation. However, even without navigation, the maximum LLD was less than $1 \mathrm{~cm}$.

Traditional navigation is currently used in $<5 \%$ of THA procedures [15], due largely to its high cost, cumbersome nature and steep learning curve. The device used in our study; however, is not associated with these drawbacks and offers a cost-effective alternative to more traditional systems. The device is compatible with all component manufacturers and has demonstrated excellent accuracy in clinical studies, most recently in large study comparing intraoperative device measurements of cup position in 356 patients with post-operative EOS imaging, where it measured anteversion to within $2.97^{\circ} \pm 4.05^{\circ}$ and inclination to within $2.17^{\circ} \pm 2.50^{\circ}$ of EOS images [31]. Previous studies have demonstrated measurement of leg length differentials within $0.3-1.3 \mathrm{~mm}$ of post-operative imaging $[18,32]$ and a low proportion (8\%) of post-operative LLDs $>5 \mathrm{~mm}$ [32], results which mirror those of our current study. Importantly, this high level of accuracy does not come at the cost of efficiency, as the device has also demonstrated time-neutrality with non-navigated THA, being associated with a time-add of 2.9 minutes [33] and a learning curve of 3-5 cases (unpublished data). This combined ease-of- use and high accuracy suggest a viable role for this technology in THA moving forward.

Despite the ongoing debate regarding its validity [34], the majority of surgeons continue to use the Lewinnek safe zone [17] as a reference orientation to minimize the risk of post-operative dislocation. Increasing, however, surgeons are taking a "functional cup position" approach to pre-operative planning. This technique involves patient-specific cup position targets, based on the patient's functional pelvic orientation during standing and sitting $[35,36]$. This is especially prevalent in cases where spinal fusion or other spinal pathologies limit the mobility of the lumbar spine and increase the workload on the hip joint itself. In our study, a functional cup position target of $40^{\circ}$ inclination and $20^{\circ}$ anteversion was set pre-operatively for each patient. Both pre- and post-operative pelvic radiographs were weightbearing and all calculations are based on functional cup orientation. In the navigation group, we noted a significantly greater proportion of cups placed within $10^{\circ}$ of this target orientation than in nonnavigated cases. Likewise, we noted a significantly higher proportion of cups within the traditional safe zone. Our findings mirror those of other studies comparing navigation to traditional methods, where cups placed with navigation assistance were between 2 and 4 times more likely to be within the safe zone [9-14]. This ability to assist the surgeon in accurately placing components in their targeted orientation is perhaps the most important benefit of navigation, and is especially important in cases with a lower margin for error such as those where hip-spine comorbidities play a critical role.

198 We noted a very low rate of complications in our study, in both the navigated and nonnavigated groups. There were no reports of device-related adverse events, including pain at the site of the iliac crest pins. While a relatively rare occurrence, there have been reports of pin-site pain with some navigation devices as high as $4 \%$ at 90 -days post-op [37]. These reports; however, have generally been recorded in studies using systems that require both iliac crest and distal femur pins, with the highest rates of pin-site pain reported in the distal femur locations [37]. Rates of pin-site pain in the iliac crest are routinely reported below $2 \%[32,38]$, and previous studies of the navigation device used in this study have similarly reported no pain at the iliac crest pin site [32]. Regarding episode of care outcomes, we did not have any cases of dislocation, revision surgery or hospital readmission in the control and intervention groups at 90 -days post-procedure. 


\section{Conclusions}

In this case-matched study, we demonstrated the ability of an imageless navigation system to improve the accuracy with which acetabular cup components are implanted during THA. The noted improvements in safe zone and LLD proportionality suggest that there is a role for such navigation systems in improving both short- and long-term outcomes in patients undergoing primary THA with posterior approach.

Table 1. Summary of Demorgraphic Data

\begin{tabular}{lccc}
\hline & Intervention (n=57) & Control (n=57) & p-value \\
\hline Gender; $\mathrm{n}(\%)$ & $28(49 \%)$ & $28(49 \%)$ & \\
Male & $29(51 \%)$ & $29(51 \%)$ & 1 \\
Female & & \\
\hline Mean age & $56.6 \pm 12.5(20-85)$ & $54.9 \pm 9.6(30-72)$ & 0.4 \\
year \pm SD (range) & $32.8 \pm 3.4(27-41)$ & $33.2 \pm 4.1(27-43)$ & 0.5 \\
\hline BMI & & & \\
\hline
\end{tabular}


Table 2: Radiographic Measurements

\begin{tabular}{|c|c|c|c|}
\hline Category & IJH & Control & p-value \\
\hline \multicolumn{4}{|l|}{ Anteversion $\left({ }^{\circ}\right)$} \\
\hline Mean value, mean (SD, range) & $20.6(9.3,1.3-40.3)$ & $25.0(11.1,2.3-46.7)$ & $0.005^{\mathrm{a}}$ \\
\hline In Lewionek SZ $\left(15 \pm 10^{\circ}\right), n / N(\%)$ & $46 / 79(58)$ & $34 / 93(37)$ & $0.005^{b}$ \\
\hline In Meftah target zone $\left(20 \pm 10^{\circ}\right), \mathrm{n} / \mathrm{N}(\%)$ & $55 / 79(70)$ & $49 / 93(53)$ & $0.02^{b}$ \\
\hline \multicolumn{4}{|l|}{ Inclination $\left({ }^{\circ}\right)$} \\
\hline Mean value, mean (SD, range) & $42.8(6.0,27.0-57.3)$ & $45.7(8.7,19.7-68.3)$ & $0.01^{a}$ \\
\hline $\begin{array}{l}\text { In Lewionek SZ and Meftal target zone } \\
\qquad\left(40 \pm 10^{\circ}\right), \mathrm{n} / \mathrm{N}(\%)\end{array}$ & $69 / 79(87)$ & $62 / 93(67)$ & 0.02 \\
\hline \multicolumn{4}{|l|}{ LLD (mm) } \\
\hline Mean value, mean (SD) & $3.1(1.5)$ & $4.6(3.4)$ & $0.36^{\mathrm{a}}$ \\
\hline$>5 \mathrm{~mm}$ LLD, $\mathrm{n} / \mathrm{N}(\%)$ & $4 / 57(7)$ & $18 / 72(31)$ & $0.09^{b}$ \\
\hline
\end{tabular}

\footnotetext{
a. t-test

b. chi-squared test
}

\section{References}

1. Masaoka T, Yamamoto K, Shishido T, et al. Study of hip joint dislocation after total hip arthroplasty. Int Orthop 2006 30(1):26-30

2. Nishii T, Sugano N, Miki H, Koyama T, Takao M, Yoshikawa H. Influence of component positions on dislocation: computed tomographic evaluations in a consecutive series of total hip arthroplasty. $\mathrm{J}$ Arthroplasty 2004 19(2):162-6

3. Oki H, Ando M, Omori H, et al. Relation between vertical orientation and stability of acetabular component in the dysplastic hip simulated by nonlinear three-dimensional finite element method. Artif Organs 2004 28(11):1050-4

4. Elkins JM, Callaghan JJ, Brown TD. The 2014 Frank Stinchfield Award: The 'landing zone' for wear and stability in total hip arthroplasty is smaller than we thought: a computational analysis. Clin Orthop Relat Res 2015 473(2):441-52

5. Kennedy JG, Rogers WB, Soffe KE, Sullivan RJ, Griffen DG, Sheehan LJ. Effect of acetabular component orientation on recurrent dislocation, pelvic osteolysis, polyethylene wear, and component migration. J Arthroplasty 1998 13(5):530-4

6. Werner BC, Brown TE. Instability after total hip arthroplasty. World J Orthop 2012. 3(8):122-30

7. Biedermann R, Tonin A, Krismer M, Rachbauer F, Eibl G, Stockl B. Reducing the risk of dislocation after total hip arthroplasty: the effect of orientation of the acetabular component. J Bone Joint Surg Br 2005 87(6):762-9

8. Barrack RL, Krempec JA, Clohisy JC, et al. Accuracy of acetabular component position in hip arthroplasty. J Bone Joint Surg Am 2013 95(19):1760-8 
9. Gurgel HM, Croci AT, Cabrita HA, Vicente JR, Leonhardt MC, Rodrigues JC. Acetabular component positioning in total hip arthroplasty with and without a computer-assisted system: a prospective, randomized and controlled study. J Arthroplasty 2014 29(1):167-71

10. Kalteis T, Handel M, Bathis H, Perlick L, Tingart M, Grifka J. Imageless navigation for insertion of the acetabular component in total hip arthroplasty: is it as accurate as CT-based navigation? J Bone Joint Surg Br 2006 88(2):163-7

11. Kalteis T, Handel M, Herold T, Perlick L, Baethis H, Grifka J. Greater accuracy in positioning of the acetabular cup by using an image-free navigation system. Int Orthop 2005 29(5):272-6

12. Lass R, Kubista B, Olischar B, Frantal S, Windhager R, Giurea A. Total hip arthroplasty using imageless computer-assisted hip navigation: a prospective randomized study. J Arthroplasty 2014 29(4):786-91

13. Lin F, Lim D, Wixson RL, Milos S, Hendrix RW, Makhsous M. Limitations of imageless computerassisted navigation for total hip arthroplasty. J Arthroplasty 2011 26(4):596-605

14. Parratte S, Argenson JN. Validation and usefulness of a computer-assisted cup-positioning system in total hip arthroplasty. A prospective, randomized, controlled study. J Bone Joint Surg Am 2007 89(3):494-9

15. Jassim SS, Benjamin-Laing H, Douglas SL, Haddad FS. Robotic and navigation systems in orthopaedic surgery: how much do our patients understand? Clin Orthop Surg 2014 6(4):462-7

16. National Joint Registry, c. 2008-2014 2016 [cited 2016 October 14]. Available from http://www.njrcentre.org.uk/njrcentre/default.aspx.

17. Lewinnek GE, Lewis JL, Tarr R, Compere CL, Zimmerman JR. Dislocations after total hipreplacement arthroplasties. J Bone Joint Surg Am 1978 60(2):217-20

18. Grosso P, Snider, M., Muir, J. M. A smart tool for intraoperative leg length targeting in total hip arthroplasty: a retrospective cohort study. The Open Orthopaedics Journal 2016 10:490-

19. Gross A, Muir JM. Identifying the procedural gap and improved methods for maintaining accuracy during total hip arthroplasty. Med Hypotheses 2016 94:93-8

20. Meftah M, Yadav A, Wong AC, Ranawat AS, Ranawat CS. A novel method for accurate and reproducible functional cup positioning in total hip arthroplasty. J Arthroplasty 2013 28(7):1200-5

21. Meermans G, Malik A, Witt J, Haddad F. Preoperative radiographic assessment of limb- length discrepancy in total hip arthroplasty. Clin Orthop Relat Res 2011 469(6):1677-82

22. Upadhyay A, York S, Macaulay W, McGrory B, Robbennolt J, Bal BS. Medical malpractice in hip and knee arthroplasty. J Arthroplasty 2007 22(6 Suppl 2):2-7

23. Danner D, Turner RH. Medical Malpractice in Revision Hip Surgery. Bono JV, McCarthy JC, Thornhill TS, Bierbaum BE, Turner RH, editors. New York: Springer; 1999.

24. Jena AB, Seabury S, Lakdawalla D, Chandra A. Malpractice risk according to physician specialty. N Engl J Med 2011 366(7):629-36

25. Konyves A, Bannister GC. The importance of leg length discrepancy after total hip arthroplasty. J Bone Joint Surg Br 2005 87(2):155-7

26. Sykes A, Hill J, Orr J, et al. Patients' perception of leg length discrepancy post total hip arthroplasty. Hip Int 2015 25(5):452-6

27. Ranawat CS, Rodriguez JA. Functional leg-length inequality following total hip arthroplasty. J Arthroplasty 1997 12(4):359-64

28. Jasty M, Webster W, Harris W. Management of limb length inequality during total hip replacement.

Clin Orthop Relat Res 1996 333:165-71

29. Snijders T, van Gaalen SM, de Gast A. Precision and accuracy of imageless navigation versus freehand implantation of total hip arthroplasty: A systematic review and meta-analysis. Int J Med Robot 2017

30. Xu K, Li YM, Zhang HF, Wang CG, Xu YQ, Li ZJ. Computer navigation in total hip arthroplasty: a meta-analysis of randomized controlled trials. Int J Surg 2014 12(5):528-33 
31. Pitta M, Ponzio D, Mayman DJ, Jerabek SA, Sculco PK. Validating the accuracy of a novel computer-assisted system using two-dimensional and three-dimensional radiographic analysis. J Arthroplasty 2018 (Submitted)

32. Paprosky WG, Muir JM, Sostak JR. Improved accuracy of component placement during total hip arthroplasty using imageless navigation: a retrospective cohort study. J Hip Surg 2018 submitted

33. Christ A, Ponzio D, Pitto M, Carroll KM, Muir JM, Sculco PK. Minimal increase in total hip arthroplasty surgical procedural time with the use of a novel surgical navigation tool. Open Orthop $\mathrm{J}$ 2018 In press

34. Abdel MP, von Roth P, Jennings MT, Hanssen AD, Pagnano MW. What Safe Zone? The Vast Majority of Dislocated THAs Are Within the Lewinnek Safe Zone for Acetabular Component Position. Clin Orthop Relat Res 2016 474(2):386-91

35. Esposito CI, Carroll KM, Sculco PK, Padgett DE, Jerabek SA, Mayman DJ. Total Hip Arthroplasty Patients With Fixed Spinopelvic Alignment Are at Higher Risk of Hip Dislocation. J Arthroplasty 2018 33(5):1449-54

36. Esposito CI, Miller TT, Kim HJ, et al. Does Degenerative Lumbar Spine Disease Influence Femoroacetabular Flexion in Patients Undergoing Total Hip Arthroplasty? Clin Orthop Relat Res 2016 474(8):1788-97

37. Newman JM, Carroll KM, Cross MB. Robotic-assisted total hip arthroplasty: a critical appraisal. Robotic Surgery: Research and Reviews 2014 1:37-42.

38. Clave A, Sauleau V, Cheval D, Williams T, Lefevre C, Stindel E. Can computer-assisted surgery help restore leg length and offset during THA? A continuous series of 321 cases. Orthop Traumatol Surg Res 2015 101(7):791-5 